\title{
Is there a role for the RNA-binding protein LARP1 in $\beta$-cells?
}

\author{
Joao Pedro Werneck-de-Castro ${ }^{1,3}$, Flavia Leticia Martins Peçanha ${ }^{1}$, Diego Silvestre ${ }^{2}$ and Ernesto \\ Bernal-Mizrachi ${ }^{1,3}$. \\ ${ }^{1}$ Division of Endocrinology, Diabetes and Metabolism, University of Miami, Miller School of Medicine, \\ Miami, Florida 33136, USA. \\ ${ }^{2}$ Institute of Nutrition Josué de Castro, Federal University of Rio de Janeiro, Rio de Janeiro, Rio de \\ Janeiro 21941090, Brazil. \\ ${ }^{3}$ Miami VA Health Care System, Miami, Florida 33136, USA.
}

\begin{abstract}
Mechanistic target of rapamycin complex 1 (mTORC1) is a cellular rheostat linking nutrient availability and growth factor to cellular protein translation. In pancreatic insulin secreting $\beta$-cells, mTORC1 deficiency or chronic hyperactivation leads to diabetes. mTORC1 complexes with Larelated protein 1 (LARP1) to specifically regulate the expression of 5 ' terminal oligopyrimidine tract (5'TOP) mRNAs which encode proteins of the translation machinery and ribosome biogenesis. We aimed to investigate the role played by LARP1 in $\beta$-cells in vivo. Here we show that LARP1 is the most expressed LARP in mouse islets and human $\beta$-cells, being 2 -4-fold more abundant than LARP1B, a member of the family that also interacts with mTORC1. Interestingly, $\beta$-cells from diabetic patients have higher LARP1 and LARP1B expression suggesting greater protein translation. These studies led us to generate a conditional LARP1 knockout mouse in $\beta$ cells ( $\beta$-Larp $1 K O$ mice). These mice exhibit normal levels of all LARP family members including Larp 1B, Larp4, Larp6 and Larp7. We did not observe any difference between control and $\beta$ Larp 1KO male mice in body weight gain, glucose levels and glucose tolerance at 8, 14 and 44 weeks of age. Female $\beta$-Larp $1 K O$ mice also performed normally during the glucose tolerance test. We then challenged the $\beta$-Larp $1 K O$ mice with high fat (HFD) or high branched-chain amino acid (BCAA) diets. During the course of 8 weeks in HFD, $\beta$-Larp $1 K O$ and control mice had similar weight gain and did not show alterations in glucose homeostasis compared to control littermates. BCAA did not impair glucose metabolism up to 8 weeks of diet challenge. However, glucose tolerance was slightly impaired in the $\beta$-Larp $1 \mathrm{KO}$ mice at 16 weeks under BCAA diet. In conclusion, LARP1 is the most abundant LARP in mouse islets and human $\beta$-cells and it is upregulated in diabetic subjects. While the lack of LARP1 specifically in $\beta$-cells did not alter glucose homeostasis in basal conditions, long-term high branched-chain amino acid feeding could impair glucose tolerance.
\end{abstract}

Email to: ebernalm@med.miami.edu

Ernesto Bernal-Mizrachi, MD, PhD

Department of Internal Medicine, Division of Endocrinology, Diabetes and Metabolism, University of Miami Miller School of Medicine, Miami, Florida, USA.

Fax: +1 (305) 2434039 


\section{INTRODUCTION}

Insulin secreting $\beta$-cell failure is a hallmark of diabetes (Alejandro, Gregg et al. 2015). Although $\beta$-cells are competent in adapting to insulin resistance by secreting more insulin and increasing in mass to maintain glucose homeostasis, the high metabolic demand will eventually progress to $\beta$ cell exhaustion and a fraction of obese patients will develop diabetes (Chang-Chen, Mullur et al. 2008, Alejandro, Gregg et al. 2015). Mechanistic target of rapamycin complex 1 (mTORC1) is a cellular rheostat linking nutrient availability and growth factor signaling to cell metabolism. We have shown previously that mTORC1 is essential to $\beta$-cell function and mass (Alejandro, Bozadjieva et al. 2017, Blandino-Rosano, Barbaresso et al. 2017). Lack of mTORC1 activity specifically in $\beta$-cells impairs proliferation and survival (Blandino-Rosano, Barbaresso et al. 2017). mTORC1 also regulates insulin processing (Blandino-Rosano, Scheys et al. 2016, Alejandro, Bozadjieva et al. 2017, Blandino-Rosano, Barbaresso et al. 2017) as well as $\beta$-cell maturation (Ni, Gu et al. 2017, Jaafar, Tran et al. 2019, Helman, Cangelosi et al. 2020). On the other hand, chronic hyperactivation of mTORC1 in $\beta$-cells culminates into $\beta$-cell dysfunction and diabetes (Bartolome, Kimura-Koyanagi et al. 2014, Ardestani, Lupse et al. 2018). These findings underscore the importance of mTORC1 signaling in $\beta$-cells.

Protein translation depends on an orchestrated assembling of proteins participating in translation initiation, ribosomal recruitment and protein elongation. mTORC1 controls cell size, proliferation, ribosomal biogenesis, protein translation and autophagy by modulating eIF4E-binding proteins (4E-BP1, 2 and 3) and ribosomal protein S6 kinases (S6K1 and 2) and ULK among others (Shimobayashi and Hall 2014, Efeyan, Comb et al. 2015). It also enhances cellular protein synthesis capacity by inducing translation of certain 5' terminal oligopyrimidine tract (5'TOP) mRNAs which encode proteins of the translation machinery and ribosome biogenesis (Jefferies, Fumagalli et al. 1997, Thoreen, Chantranupong et al. 2012, Meyuhas and Kahan 2015, Hinnebusch, Ivanov et al. 2016). mTORC1 inhibition by rapamycin represses TOP mRNA translation (Jefferies, Reinhard et al. 1994, Terada, Patel et al. 1994, Jefferies, Fumagalli et al. 1997) and the 4EBP proteins have been proposed as suppressors of TOP mRNAs translation (Thoreen, Chantranupong et al. 2012). Recently, a downstream target of mTORC1, the La-related protein 1 (LARP1), also known as RNA-binding protein LARP1, has been described as part of the protein complex regulating the 5'-TOP mRNA translation (Tcherkezian, Cargnello et al. 2014, Deragon and Bousquet-Antonelli 2015, Fonseca, Zakaria et al. 2015, Mura, Hopkins et al. 2015, Hong, Freeberg et al. 2017, Lahr, Fonseca et al. 2017). The LARP family consists of six members: LARP1, 2 (1B), 4, 5 (4B), 6, and 7 (Bousquet-Antonelli and Deragon 2009, Hong, Freeberg et al. 2017). They all contain the RNA-binding domain but only LARP 1 and LARP1B present the DM15 motif and interact with mTORC1 (Bousquet-Antonelli and Deragon 2009, Hong, Freeberg et al. 2017).

The role of LARP1 in protein synthesis and mTORC1-mediated TOP mRNA translation is controversial. Studies in HeLa and HEK293 cells have demonstrated that LARP1 positively regulates protein synthesis (Burrows, Abd Latip et al. 2010, Aoki, Adachi et al. 2013, Tcherkezian, Cargnello et al. 2014). Knockdown of LARP1 impaired cell division and migration, and induces cell apoptosis as well as a $15 \%$ drop in overall protein synthesis accompanied by hypophosphorylated 4E-BP1 levels (Burrows, Abd Latip et al. 2010), indicating participation in cap-dependent translation. LARP1 depletion was associated with a decreased TOP mRNAs 
translation (Tcherkezian, Cargnello et al. 2014). In contrast, also using HEK293T and HeLa cells, Fonseca et al. concluded that LARP1 functions as an important repressor of TOP mRNA translation downstream of mTORC1 (Fonseca, Zakaria et al. 2015). They showed that LARP1 interacts with TOP mRNAs in an mTORC1-dependent manner and competes with the eIF4G for TOP mRNA binding. Reducing LARP1 protein levels by siRNA attenuates the inhibitory effect of rapamycin and Torin 1 on TOP mRNA translation (Fonseca, Zakaria et al. 2015). That LARP1 directly binds the cap and adjacent 5'TOP motif of TOP mRNAs impeding access of eIF4E and eIF4F assembly was confirmed later (Lahr, Fonseca et al. 2017). Phosphorylation of LARP1 by mTORC1 and Akt/S6K1 dissociates it from 5'UTRs and relieves its inhibitory activity (Hong, Freeberg et al. 2017). Concomitantly, phosphorylated LARP1 scaffolds mTORC1 on the 3'UTRs to facilitate mTORC1-dependent induction of translation initiation. Although LARP1 has inhibitory effects on TOP mRNA translation, LARP1 loss of function causes inefficient translation elongation (Hong, Freeberg et al. 2017). The in vivo role of LARP proteins has been limited by the lack of animal models to study the tissue specific importance of this molecule.

We document herein that LARP1 is the most abundant of the family in human $\beta$-cells and mouse islets by our own experiments and by assessing public RNA expression databases. Interestingly, diabetes increases LARP1 and LARP1B in human $\beta$-cells, suggesting higher protein translation capacity and consistence with greater mTORC1 activity in diabetes. To study the role of LARP1 in $\beta$-cells, we developed mice with conditional inactivation in pancreatic $\beta$-cells. We found that Larpl gene disruption in mouse $\beta$-cells is dispensable for glucose homeostasis during normal conditions or after administration of high fat. Challenging the $\beta$-Larp $1 K O$ mice with high branched-chain amino acid diets only impaired glucose tolerance after 16 weeks. We conclude that although LARP1 is highly expressed in $\beta$-cells and is up-regulated in diabetogenic conditions, yet it is not essential for glucose homeostasis in normal conditions. LARP1 could play a role in longterm high branched-chain amino acid feeding.

\section{METHODS}

\section{Human $\beta$-cells gene expression database}

In order to determine LARP1 family gene expression in human $\beta$-cells, we assessed publicly available databases (Blodgett, Nowosielska et al. 2015, Segerstolpe, Palasantza et al. 2016). LARP family gene expression in human fetal and adult $\beta$-cells were calculated from Blodgett et al. (Blodgett, Nowosielska et al. 2015). In addition, single-cell transcriptome of healthy and type 2 diabetic subjects' database by Segerstolpe et al. (Segerstolpe, Palasantza et al. 2016) was used to assess the expression of LARP family members in $\beta$-cells.

\section{Mice}

All the procedures were approved by University of Miami IACUC committee (IACUC protocol \#18-168). To generate the floxed-Larp1 mouse, embryonic stem cells containing the floxed Larp1 construct (Fig 3A) were obtained from the International Mouse Phenotype Consortium

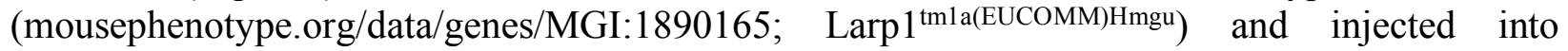
blastocyst to generate chimeric mice by the University of Michigan Transgenic Animal Model core (Alejandro, Bozadjieva et al. 2017). After identifying germline transmission, founder lines were selected and bred into $C 57 B L / 6 J$ background. In order to obtain the LARP1 knockout mice specifically in $\beta$-cells $(\beta$-Larp1KO), floxed-Larpl mice were crossed with mice expressing cre- 
recombinase under the rat insulin promoter activity $\left(R I P-C r e^{H e r r}\right.$ mice (Alejandro, Bozadjieva et al. 2017, Blandino-Rosano, Barbaresso et al. 2017) . We also disrupted Larpl gene by crossing the Floxed-Larp1 mice with the Ins1-cre mouse (B6(Cg)-Ins1tm1.1(cre)Thor/J; Jackson's lab stock no:026801).

Metabolic studies. Blood glucose levels were determined from blood obtained from the tail vein using ACCU-CHEK II glucometer (Roche). Glucose tolerance test was performed in $6 \mathrm{~h}$ fasted animals by injecting glucose intraperitoneally $(2 \mathrm{~g} / \mathrm{kg})$.

\section{Diets}

High Fat Diet (HFD; cat no D12492; $20 \%$ of carbohydrate, $20 \%$ protein and $60 \%$ fat; Research Diets, New Brunswick, NJ) and Branched-chain amino acid enriched diet (BCAA; cat no D07010503; $67 \%$ of carbohydrate, $23 \%$ protein and 10\% fat Research Diets). The BCAA has $150 \%$ more leucine, isoleucine and valine concentrations. Control standard diet contains $55 \%$ of carbohydrate, $23 \%$ protein and $22 \%$ fat (ENVIGO).

\section{Pancreatic islets isolation and quantitative real-time PCR}

Islets were isolated by collagenase digestion method as detailed recently (Werneck-de-Castro, Blandino-Rosano et al. 2020). After overnight recover in non-treated plastic petri dishes (VWR) containing RPMI 1640 (Corning cellgro) supplemented with 10\% FBS, 1\% Penicillin and Streptomicin and $5.5 \mathrm{mM}$ glucose, total RNA was isolated using RNeasy (Qiagen) from 80-100 islets. cDNA was synthesized from 0.5 ug of total RNA using random hexamers and was reverse transcribed using Superscript II (High Capacity cDNA reverse transcription kit; Applied biosystems). Real-time PCR was performed on an ABI 7000 sequence detection system using POWER SYBR-Green PCR Master MIX (Applied Biosystems). Primers were purchased from IDT Technologies and sequences are shown in Table 1.

\section{Statistics}

Data are presented as mean \pm sem. Student $t$ test was employed to assess statistical difference between means of two groups in one time point, e.g. control vs diabetes (Figure 2C) and control vs $\beta$-Larp $1 K O$ mice (Fig 3B). One-way analysis of variance (ANOVA) followed by Dunnet's posthoc test was performed to compare LARP family gene expression to the LARP1 mRNA levels (Fig 2A and B). Two-way ANOVA followed by Tukey's posthoc test was used to identify differences between control vs $\beta$-LarplKO mice over time, e.g. body weight gain (Fig 4A, 6A and 7A) and glucose levels during intraperitoneal and oral glucose tolerance test. Results were considered statistically significant when the $\mathrm{p}$ value was less than 0.05 .

\section{RESULTS}

LARP1 is the most expressed LARP in human and mouse pancreatic islets and $\beta$-cells

The RNA-binding La-related protein (LARP) family consists of six members namely LARP1, 2 (1B), 4, 5 (4B), 6, and 7 (Bousquet-Antonelli and Deragon 2009, Hong, Freeberg et al. 2017). LARP1 and LARP1B share a common DM15 motif and are mTORC1 targets. We first measured the mRNA levels of the LARP family in isolated mouse islets (Fig 1A). Remarkably, LARP1 is the most expressed LARP in mouse islets, being 4-5-fold higher than LARP1B, LARP4 and LARP7 (Fig 1A). LARP6 is barely detectable, beeing only 5\% of LARP1 expression (Fig 1A). 
Assessment of publicly available mRNA transcription databases for human $\beta$-cells (Blodgett, Nowosielska et al. 2015, Segerstolpe, Palasantza et al. 2016) shows that fetal and adult purified $\beta$ cells present higher levels of LARP1 mRNA levels ( 2-fold) compared to its paralog gene LARP1B (Fig 1B and C; (Blodgett, Nowosielska et al. 2015)). Single-cell transcriptome profiling of human $\beta$-cells in healthy subjects and type 2 diabetes (T2D) further corroborates that LARP1 is the most abundant LARP in $\beta$-cells (Fig 2A) with similar pattern seen in T2D (Fig 2B). Interestingly, diabetes increases LARP1 ( 30\%) and LARP1B (100\%) (Fig 2C), suggesting higher protein translation capacity, consistent with greater mTORC1 activity in diabetes (Ardestani, Lupse et al. 2018).

\section{B-Larp1KO mice: in vivo model to assess LARP1 function}

Whereas the role played by mTORC1 in $\beta$ - and $\alpha$-cells was recently revealed (Alejandro, Bozadjieva et al. 2017, Blandino-Rosano, Barbaresso et al. 2017, Bozadjieva, Blandino-Rosano et al. 2017, Ni, Gu et al. 2017, Sinagoga, Stone et al. 2017), the role of the mTORC1 target LARP1 on pancreatic $\beta$-cells has never been studied. mTORC1 complexes and phosphorylates LARP1, but whether LARP1 inhibits or stimulates mTORC1-mediated protein translation of TOP mRNAs is still under debate (Burrows, Abd Latip et al. 2010, Aoki, Adachi et al. 2013, Tcherkezian, Cargnello et al. 2014, Fonseca, Zakaria et al. 2015, Mura, Hopkins et al. 2015, Hong, Freeberg et al. 2017, Lahr, Fonseca et al. 2017). Published work has characterized the role of LARP1 using in vitro models. Therefore, we decided to generate a LARP1 deficient mouse specifically in $\beta$-cells to evaluate LARP1 function in the context of $\beta$-cell biology. We generated a floxed-LARP1 mouse with the lox $\mathrm{p}$ sequence flanking exon 4 (Figure 3A). Then, floxed-LARP1 mice were crossed initially with mice expressing Cre recombinase under the control of the rat insulin promoter to generate the $\beta$-Larp $1 K O$ mouse. LARP1 mRNA levels decreased $80 \%$ in isolated islets of the $\beta$ Larp 1KO compared to littermate control mice (Fig 3B). The remaining expression is probably from non- $\beta$-cells (e.g. $\alpha$ and $\delta$ cells) and acinar contaminants. The expected recombination in $\beta$ cells with the RIP-cre mouse is about 90-95\% (Blandino-Rosano, Barbaresso et al. 2017), therefore, the residual expression can also be explained by the non-recombined $\beta$-cells. LARP1 and LARP1B are very similar in structure sharing the DM15 region, known as LARP1 motif (Stavraka and Blagden 2015, Hong, Freeberg et al. 2017). Therefore, we measured LARP1B and other LARP family members mRNA expression in isolated islet. The deficiency is specific to LARP1, since the $\beta$-Larp $1 K O$ mice have normal expression of LARP1B, LARP4 and LARP7. LARP6 mRNA levels tended to be increased in the $\beta$-Larp1KO mice but did not reach statistical significance (Fig 3B).

\section{$\beta$-Larp1KO mice grow and age normally and exhibit normal glucose homeostasis}

We followed up glucose metabolism in $\beta$-Larp $1 K O$ male mice at different ages (Fig 4). Body weight, fed and fasting glycemia, and tolerance to intraperitoneal glucose load were similar in the first cohort of animals at 4 and 8 weeks of age (Fig 4A-C). Using a second cohort of $\beta$-Larp 1 KO male mice, no difference in body weight, fed and fasting glycemia and glucose tolerance test was observed at 14 weeks of age (Fig 4D-F). In addition, there was no difference in glucose tolerance in $\beta$-Larp $1 K O$ female mice (Fig 4G). We also crossed the floxed-LARPl mouse with Ins 1-Cre knock-in mice (Ins1-cre) and found no difference in glucose tolerance (Fig 4H) despite the 80\% reduction in Larp1 expression by islets (Fig 4I). To investigate the effects of aging and the lack of LARP1 in $\beta$-cells, glucose tolerance test was performed in a third cohort of mice aged until almost 
a year (Fig 5). Aged $\beta$-Larp $1 K O$ male mice performed similarly to control mice in the glucose tolerance test and weighted equally at 44 weeks of age (Fig 5A and B).

\section{Exposure of $\beta$-Larp1KO mice to high fat diet did not alter glucose homeostasis}

It is well-characterized that high fat diet (HFD) provokes insulin resistance, increasing the demand for insulin production and secretion by $\beta$-cells, resulting in higher insulinemia. Insulin in turn stimulates $\beta$-cell expansion through the activation of Akt/mTORC1 pathway. Therefore, we decided to challenge the $\beta$-Larp $1 K O$ mice under HFD. We placed the first cohort used in Fig 4A under HFD. The $\beta$-Larp 1KO mice gained weigh as much as control mice (Fig 6A and B). There was no difference in fed glycemia before, during and after 8 weeks under HFD (Fig 6C). Intraperitoneal glucose tolerance test at 4 and 8 weeks after HFD was comparable between $\beta$ Larp 1KO and control mice (Fig 6D and E). We also tested whether incretins could play a role in the $\beta$-Larp $1 K O$ mice by performing an oral glucose tolerance test and found no difference between the groups (Fig 6F).

\section{Long-term exposure to high branched-chain amino acid diet slightly impairs glucose tolerance in $\beta$-Larp1KO mice.}

As an alternative method, we placed the second-cohort used in Fig 4D and E under branched-chain amino acids diet (BCAA). BCAA diet directly stimulate mTORC1 activity, especially the enriched L-leucine amino acid (Condon and Sabatini 2019). There was no difference in body weight gain in the $\beta$-Larp $1 K O$ and control groups (Fig 7A and B). Glucose tolerance was the same between the groups at 4 and 8 weeks after high BCAA diet (Fig 7C and D). However, after 16 weeks, $\beta$ Larp $1 K O$ were slightly intolerant to glucose compared to littermate control mice (Fig $7 \mathrm{E}$ and $\mathrm{F}$ ). Oral tolerance to glucose was the same after 17 weeks in BCAA diet (Fig 7G).

\section{DISCUSSION}

We report herein that La related protein 1 (LARP1) is highly expressed in human $\beta$-cells and mouse islets compared to the other members of the family. Furthermore, type 2 diabetes upregulates LARP1 and LARP1B consistent with high protein translation, cell proliferation and mTORC1 activity (Yuan, Rafizadeh et al. 2017, Ardestani, Lupse et al. 2018). However, mice lacking LARP1 specifically in $\beta$-cells ( $\beta$-Larp1KO mice) developed normally and glucose metabolism was similar to control mice. Even under diet-induced stress (high fat or diet high in branched-chain amino acid), glucose metabolism in $\beta$-Larp $1 \mathrm{KO}$ mice did not deviate from control mice. There was only a minor impaired glucose tolerance after long-term (16 weeks) in BCAA diet. Therefore, we conclude that LARP1 is dispensable for pancreatic $\beta$-cell function and glucose homeostasis.

A constant cellular regulation of protein synthesis and breakdown determine cellular function and growth. During the progression of diabetes, $\beta$-cells expand in size and number to meet the high metabolic demand imposed by insulin resistance (Alejandro, Gregg et al. 2015). mTORC1 signaling pathway is highly activated in $\beta$-cells from diabetic patients and rodents, indicating enhanced protein synthesis and increased proliferation (Yuan, Rafizadeh et al. 2017). The current studies were designed to interrogate the in vivo role of LARP1 in $\beta$-cell function based on previous finding that LARP1 and mTORC1 work together to regulate the translation of key mRNAs, the 5' 
TOP mRNA (Hong, Freeberg et al. 2017, Lahr, Fonseca et al. 2017, Philippe, Vasseur et al. 2018). This group of mRNA encodes components of the protein translation machinery. We found that LARP1 is the most expressed LARP in mouse islets and human $\beta$-cells. Moreover, LARP1 is upregulated in $\beta$-cells in patients diagnosed with type 2 diabetes. This is consistent with the in vitro findings that LARP1 regulates proliferation (Tcherkezian, Cargnello et al. 2014, Hong, Freeberg et al. 2017, Berman, Thoreen et al. 2020). In several different types of cancer, highly phosphorylated LARP1 has been reported, although kinases other than mTORC1 are likely to induce LARP1 phosphorylation in cancer (Hopkins, Mura et al. 2016, Ye, Lin et al. 2016, Xu, Xu et al. 2017, Berman, Thoreen et al. 2020).

Surprisingly, we found that $\beta$-LarplKO mice exhibit a normal phenotype. mTORC1 plays a fundamental role in $\beta$-cell physiology by controlling 5 ' cap-dependent translation of critical proteins in $\beta$-cell (Blandino-Rosano, Barbaresso et al. 2017, Ni, Gu et al. 2017). mTORC1 activity in $\beta$-cells is higher during embryological development and in the first week of post-natal maturation, followed by lower activity levels in mature $\beta$-cells (Ni, Gu et al. 2017, Sinagoga, Stone et al. 2017, Jaafar, Tran et al. 2019, Helman, Cangelosi et al. 2020, Katsumoto and Grapin-Botton 2020). mTORC1 is reactivated in diabetic states and the chronic hyperactivation could play a role in $\beta$-cells dysfunction or failure (Yuan, Rafizadeh et al. 2017, Ardestani, Lupse et al. 2018). The fact that $\mathrm{mTORC} 1$ and LARP1 interact to each other to control cellular protein translation capacity, and, more importantly, that mTORC1 activity and LARP1 expression are both increased in diabetes prompted us to generate a conditional mouse strain to disrupt LARP1 specifically in $\beta$ cells. The reasons for the normal glucose metabolism in the $\beta$-Larp $1 K O$ mice are not clear. We observed an $80 \%$ reduction of Larpl gene expression in islets of $\beta$-Larp $1 K O$ mice. This is similar to the $80 \%$ reduction in mTORC1 signaling found in the $\beta$-RaptorKO mice generated by crossing the floxed-raptor mouse with the same RIP-Cre mouse used to produce the $\beta$-LarplKO mice (Blandino-Rosano, Barbaresso et al. 2017). The RIP-Cre-induced recombination is $\sim 90-95 \%$ of all insulin positive cells (Alejandro, Bozadjieva et al. 2017, Blandino-Rosano, Barbaresso et al. 2017). We speculate that the remaining $20 \%$ expression in mouse islets is coming from non- $\beta$-cells, very few cells scaping from recombination and minor acinar contamination of the islet preparation. Due to ectopic cre-recombinase expression outside the pancreas in the RIP-cre mice, we confirmed the neutral metabolic phenotype in the $\beta$-Larp1KO mice by using the Ins 1-Cre mouse. Therefore, it is unlikely that normal $\beta$-cell function in the $\beta$-Larp $1 K O$ mice is due to poor cre-mediated recombination.

An alternative explanation to the lack of phenotype in the $\beta$-Larp $1 K O$ mice would be an upregulation of other members of the family. The LARP family consists of six members: LARP1, 2 (1B), 4, 5 (4B), 6, and 7 (Bousquet-Antonelli and Deragon 2009, Hong, Freeberg et al. 2017). They all contain the RNA-binding domain but only LARP1 and LARP1B present the DM15 motif and interact with mTORC1 (Bousquet-Antonelli and Deragon 2009, Hong, Freeberg et al. 2017). In the $\beta$-Larp $1 K O$ mice, islet LARP1B expression was similar to control littermates. Although there was trend to higher levels of LARP6 in $\beta$-Larp1KO mice, this is unlikely to explain the normal phenotype as LARP6 is barely expressed in mouse islets $(<5 \%)$ and even a 3-fold induction would still result in very low levels of LARP6. However, it is possible that expression of other LARP family members at normal or slightly increase levels (LARP6) is sufficient to maintain protein translation and $\beta$-cell function. 
The small increase in glucose levels observed in the intraperitoneal glucose tolerance test in the $\beta$ Larp 1KO mice undergoing long-term high BCAA diet (16 weeks) opens the possibility that the lack of LARP1 potentially limits protein synthesis in prolonged and sustained mTORC1 activation as in diabetogenic conditions. However, the upregulation of LARP1 in diabetes (Fig 1B) might attenuate the absence of LARP1 in the responses to HFD or BCAA diet.

In summary, LARP1 is highly expressed in human $\beta$-cells and mouse islets, and is upregulated in diabetes. However, LARP1 is dispensable for pancreatic $\beta$-cell function and glucose homeostasis in vivo. $\beta$-cell adaptation to chronic exposure to high concentration of branched-chain amino acids may require LARP1.

\section{ACKNOWLEDGEMENTS}

The authors declare no conflict of interest. We would like to appreciate the technical support provided by Portia Ritter and Pau Romaguera Llacer. The current studies were funded by the National Institute of Health (NIDDK) grant R01-DK073716 and DK084236. 
Table 1. qRT-PCR primer sequences.

\begin{tabular}{lc} 
Gene name & Sequence \\
\hline Larp1_Forward & CAAAAGTGTGCAGCCACAGTC \\
Larp1_Reverse & CCCATTCTTTTCCTCCCCCG \\
Larp1B_Forward & TCCCATACAGTCAGGGTGATGA \\
Larp1B_Reverse & CCAACCTTCTCCACAAGGGG \\
Larp4_Forward & CCTGCAGGAACCTCGAAAGT \\
Larp4_Reverse & TGGTTTCTCATGCGGCTTCT \\
Larp6_Forward & TTCAAGAAGGTGAAACACCTCAC \\
Larp6_Reverse & GTCCTCGTTCAACTCCAGGG \\
Larp7_Forward & TGGGCGAGGAGGTTATACCA \\
Larp7_Reverse & GCCTGCTGTAGGCGCTTTA \\
INS1_Forward & CACCCCACCTGGAGACCTTA \\
INS1_Reverse & TGAAACAATGACCTGCTTGCTG \\
INS2_Forward & GCAAGCAGGAAGGTTATTGTTTCA \\
INS2_Reverse & GCTTGACAAAAGCCTGGGTG \\
CycloB_Forward & GGAGATGGCACAGGAGGAA \\
CycloB_Reverse & GCCCGTAGTGCTTCAGCTT \\
\hline
\end{tabular}




\section{FIGURES}
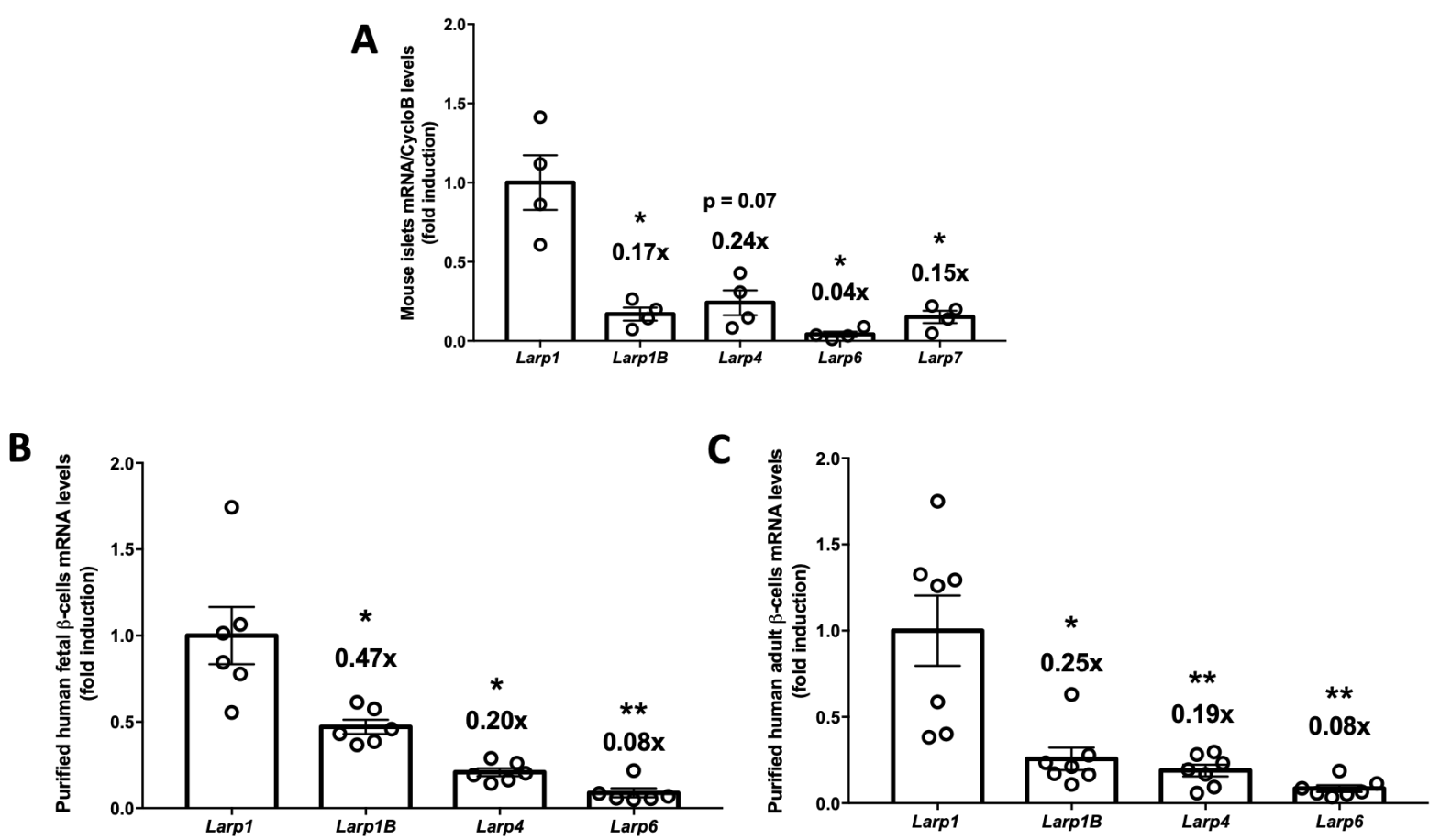

Figure 1. LARP1 is the most expressed LARP in mouse islets and human $\boldsymbol{\beta}$-cells. (A) LARP family mRNA levels in isolated mouse islets, (B) LARP family gene expression in human fetal $\beta$ cells and (C) Same as in B except that adult human $\beta$-cells were used $* p<0.05, * * p<0.01$ and $* * * \mathrm{p}<0.001$ compared to LARP1 mRNA levels assessed by one-way analysis of variance $(A N O V A)$ followed by Dunnet's posthoc test. Numbers on top of the bars denote fold reduction. Figures $\mathrm{B}$ and $\mathrm{C}$ are analysis of RNA sequencing publicly available database by Blodgett et al. (Blodgett, Nowosielska et al. 2015). 

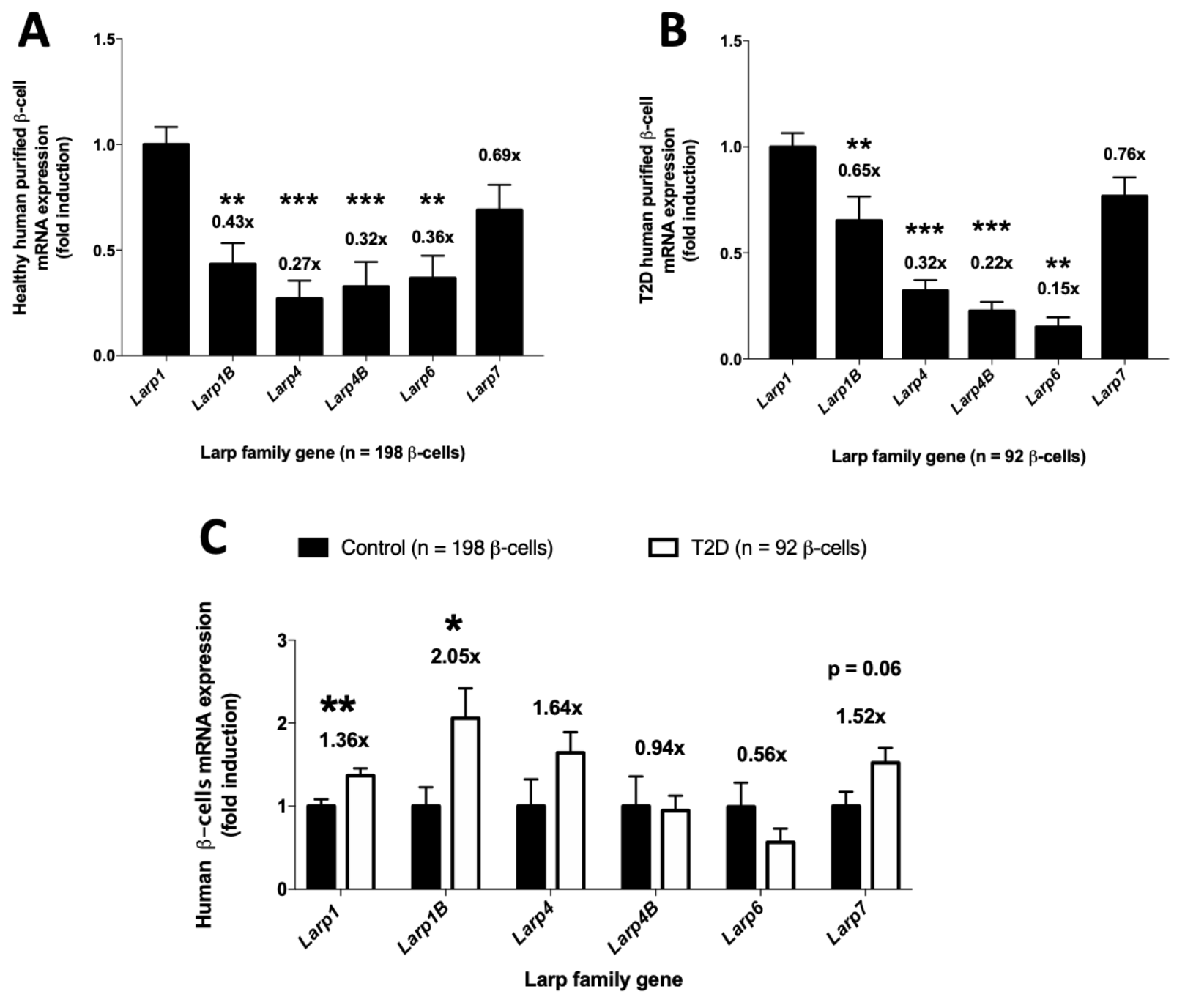

Figure 2. Diabetes increases Larp1 and Larp1B expression in human $\beta$-cells. (A) LARP family mRNA levels in human $\beta$-cells of healthy subjects. (B) Same as in A except that human $\beta$-cells from type 2 diabetic patients were used. $* * \mathrm{p}<0.01$ and $* * * \mathrm{p}<0.001$ compared to LARP1 mRNA levels assessed by one-way analysis of variance $(A N O V A)$ followed by Dunnet's posthoc test. (C) Fold induction of LARP family mRNA levels in $\beta$-cells from type 2 diabetes compared to healthy control subjects shown in A and B. $* \mathrm{p}<0.05$ and $* * \mathrm{p}<0.01$ compared to healthy subjects assessed by Student's T test. Numbers on top of the bars denote fold induction. All data were obtained by analysis of single-cell transcriptome available by Segerstolpe et al. (Segerstolpe, Palasantza et al. 2016) 
A
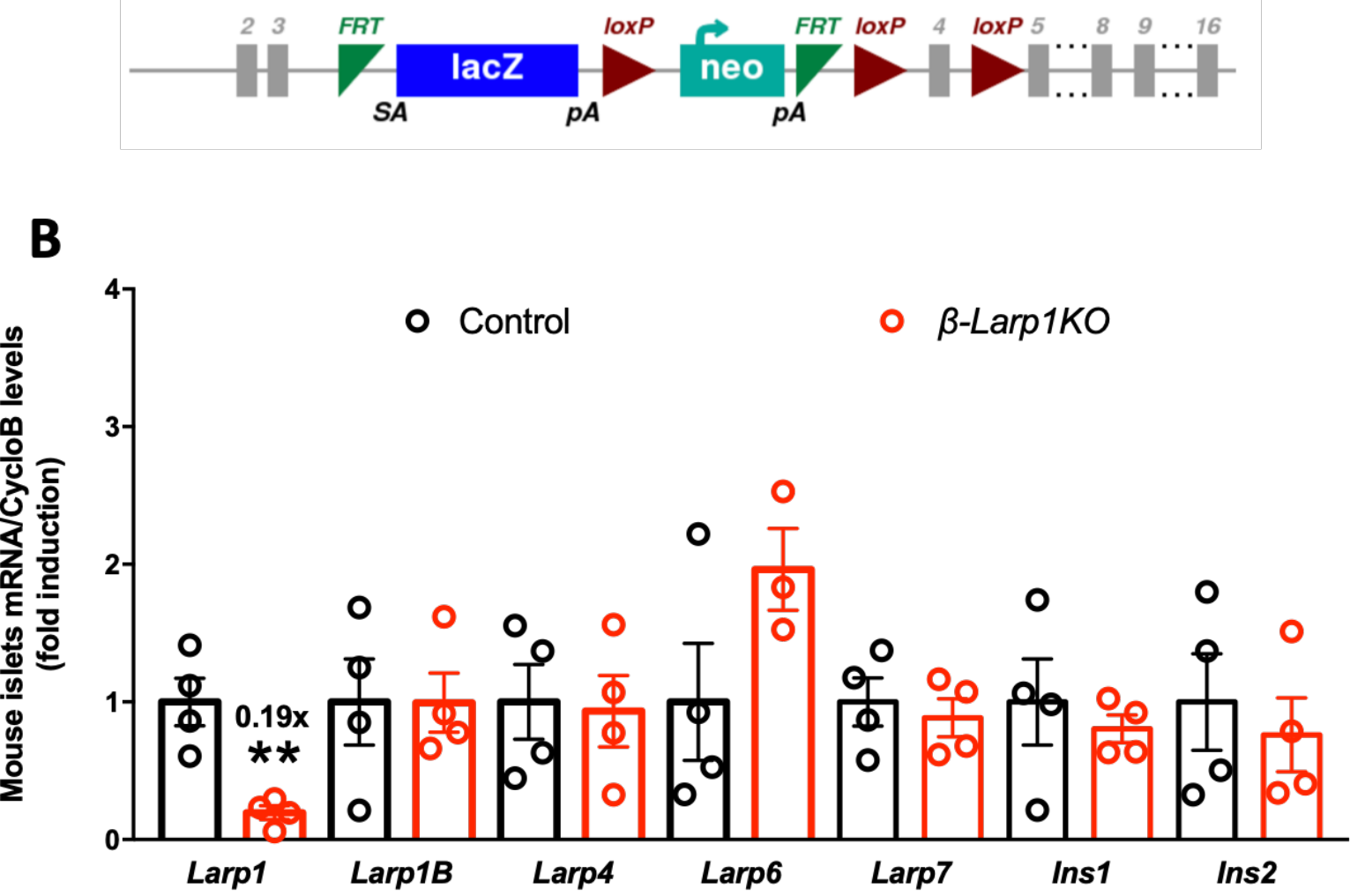

Figure 3. Generation of conditional knockout mice in $\beta$-cells ( $\beta$-Larp1KO). (A) Larp1 gene construct used to generate the floxed-Larp1 mouse. Cre-recombinase target Loxp sequences were inserted flanking exon 4. (B) LARP family and insulin mRNA levels in isolated islets from control and $\beta$-Larp $1 K O$ mice. ** $\mathrm{p}<0.01$ compared to control mice assessed by Student's T test. Number on top of Larpl bar denote fold induction. 

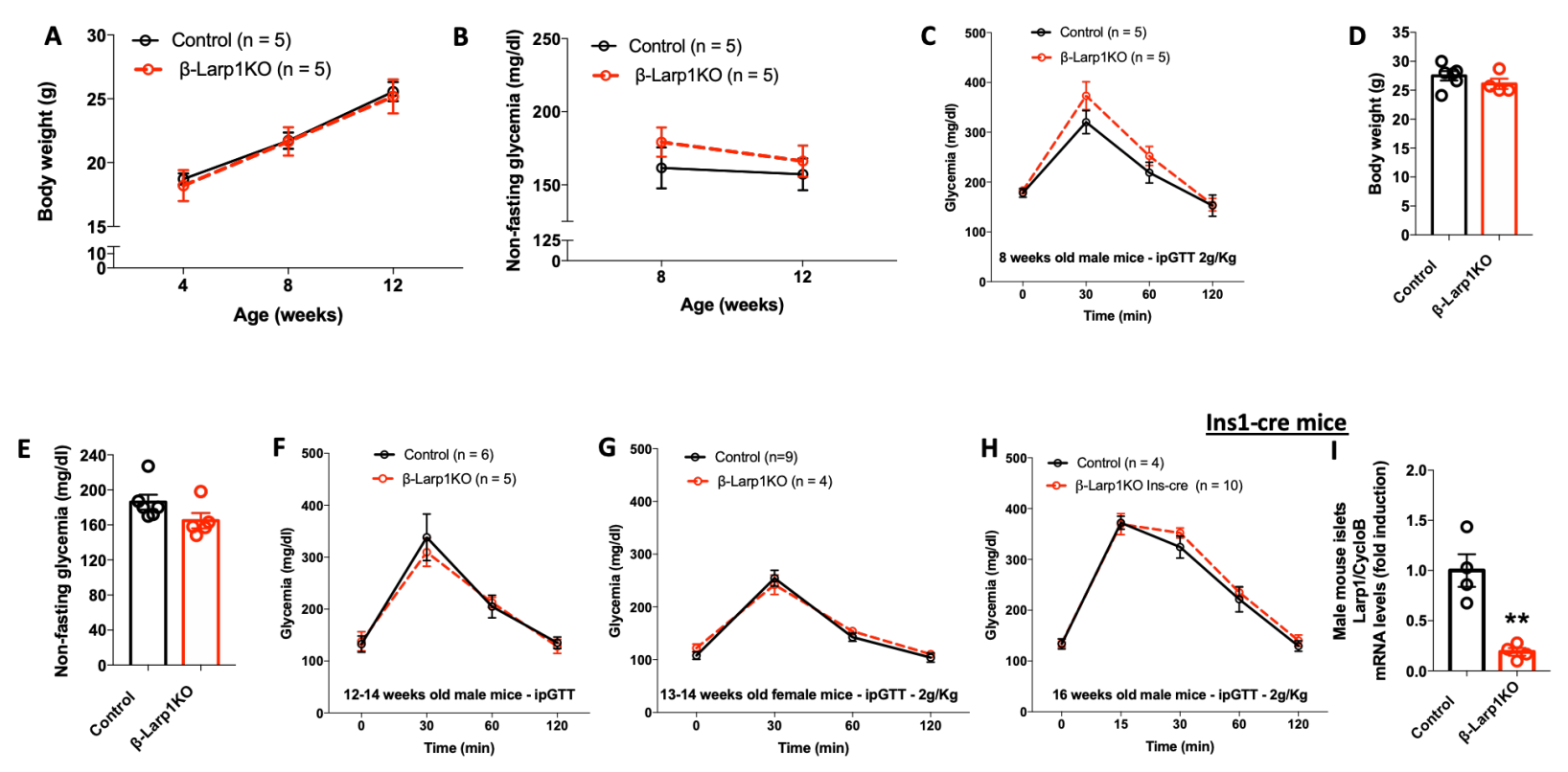

Figure 4. Males and females $\beta$-Larp1KO mice have normal glucose homeostasis. (A) Body weight gain, (B) fed glycemia and (C) intraperitoneal glucose tolerance test (ipGTT) in the first cohort of male $\beta$-Larp1KO mice; (D) Body weight, (E) fed glycemia and (F) ipGTT in the second cohort of male $\beta$-LarplKO mice; (G) ipGTT in female $\beta$-Larp $1 K O$ mice and (H) ipGTT in male $\beta$-Larp 1KO mice generated by crossing the floxed-Larp1 mice with the Ins1-cre mice instead of Rip-Cre used in A-G. ** $\mathrm{p}<0.01$ compared to control mice assessed by Student's T test. 

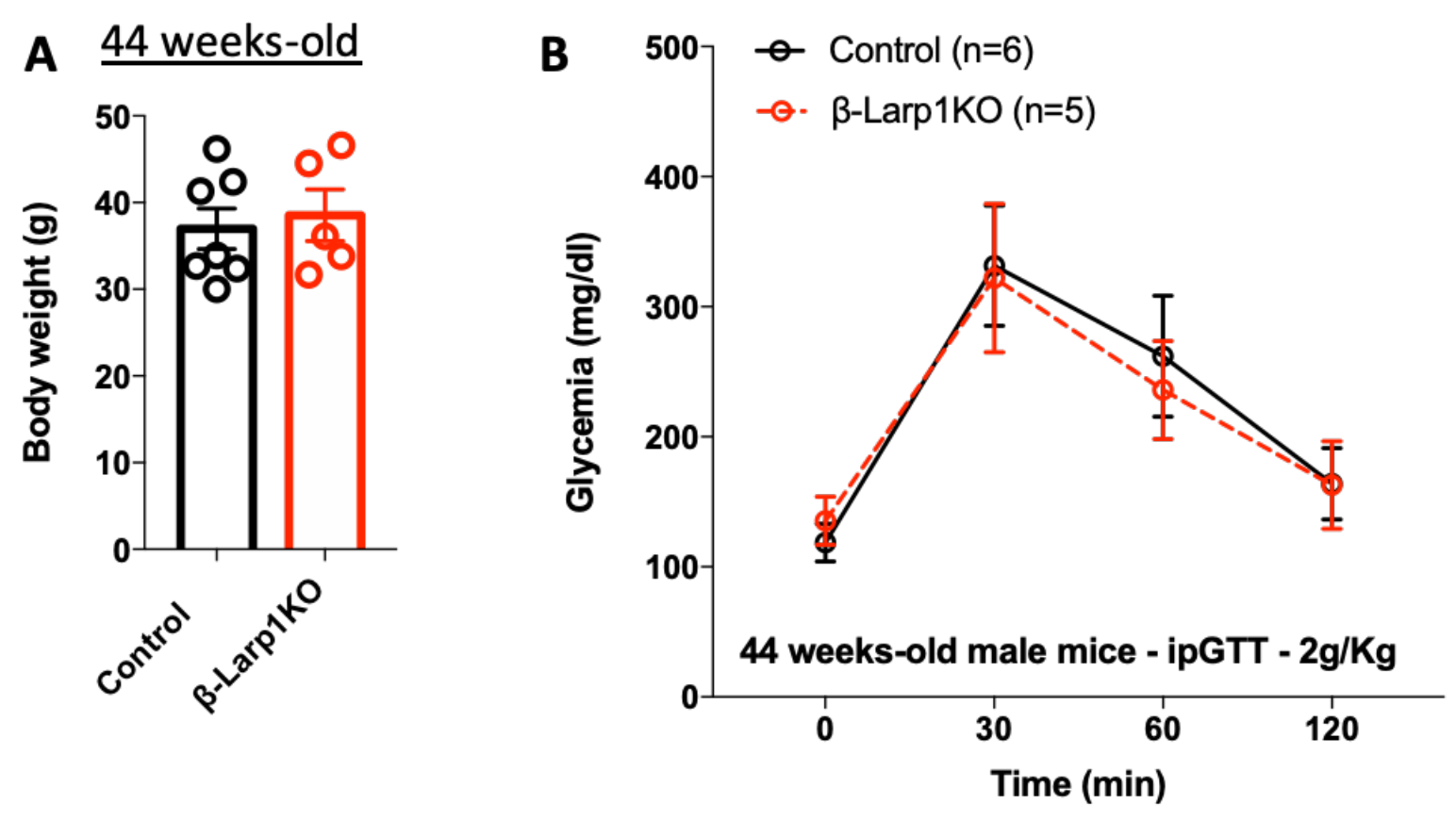

Figure 5. Aged $\beta$-Larp1KO mice are tolerant to glucose as control mice. (A) Body weight and (B) intraperitoneal glucose tolerance test in male $\beta$-Larp $1 K O$ mice at 44 weeks of age. 

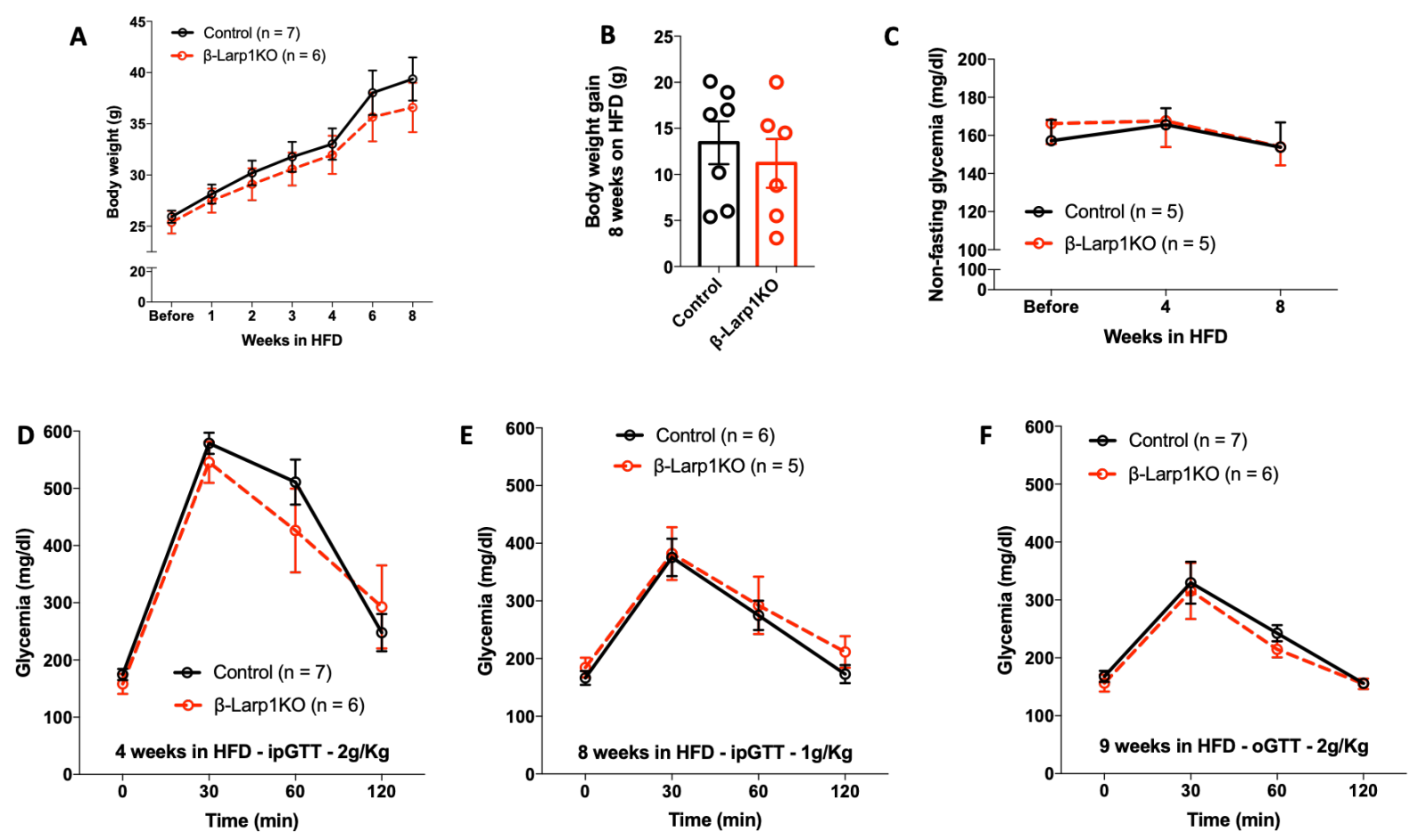

Figure 6. $\beta$-Larp1KO mice under high fat diet. (A) Body weight, (B) Body weight gain and (C) fed glycemia of mice fed with high fat diet ( $60 \%$ fat) for 8 weeks. Intraperitoneal glucose tolerance test (ipGTT) after (D) 4 and (E) 8 weeks in HFD. (G) Oral glucose tolerance test (ipGTT) after 9 weeks in HFD. 

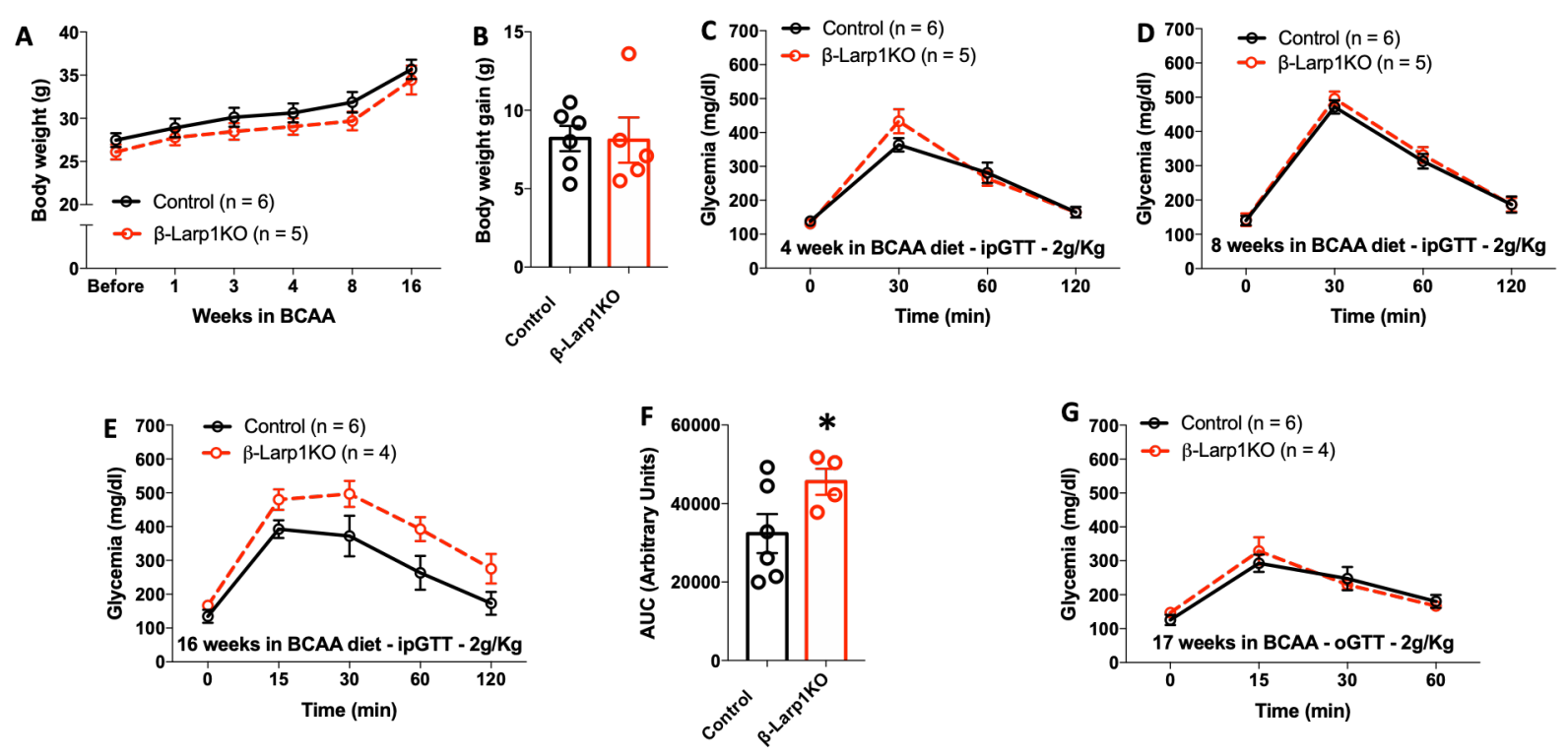

Figure 7. $\beta$-Larp1KO mice under high branched-chain amino acid diet (BCAA). (A) Body weight and (B) Body weight gain of mice fed with high branched-chain aminoacid diet (150\% BCAA) for 16 weeks. (C) Intraperitoneal glucose tolerance test (ipGTT) after 4 (D) 8 and (E) 16 weeks in BCAA diet. (F) Quantification of the area under the curve in the ipGTT performed in E. (G) Oral glucose tolerance test (ipGTT) after 17 weeks in BCAA diet. * p $<0.05$ compared to control mice assessed by Student's T test. 


\section{REFERENCES}

Alejandro, E. U., N. Bozadjieva, M. Blandino-Rosano, M. A. Wasan, L. Elghazi, S. Vadrevu, L. Satin and E. Bernal-Mizrachi (2017). "Overexpression of Kinase-Dead mTOR Impairs Glucose Homeostasis by Regulating Insulin Secretion and Not beta-Cell Mass." Diabetes 66(8): 2150-2162.

Alejandro, E. U., B. Gregg, M. Blandino-Rosano, C. Cras-Meneur and E. Bernal-Mizrachi (2015). "Natural history of beta-cell adaptation and failure in type 2 diabetes." Mol Aspects Med 42: $19-41$.

Aoki, K., S. Adachi, M. Homoto, H. Kusano, K. Koike and T. Natsume (2013). "LARP1 specifically recognizes the 3' terminus of poly(A) mRNA." FEBS Lett 587(14): 2173-2178.

Ardestani, A., B. Lupse, Y. Kido, G. Leibowitz and K. Maedler (2018). "mTORC1 Signaling: A DoubleEdged Sword in Diabetic beta Cells." Cell Metab 27(2): 314-331.

Bartolome, A., M. Kimura-Koyanagi, S. Asahara, C. Guillen, H. Inoue, K. Teruyama, S. Shimizu, A. Kanno, A. Garcia-Aguilar, M. Koike, Y. Uchiyama, M. Benito, T. Noda and Y. Kido (2014). "Pancreatic beta-cell failure mediated by mTORC1 hyperactivity and autophagic impairment." Diabetes 63(9): 2996-3008.

Berman, A. J., C. C. Thoreen, Z. Dedeic, J. Chettle, P. P. Roux and B. P. Sarah (2020). "Controversies around the function of LARP1." RNA Biol: 1-11.

Blandino-Rosano, M., R. Barbaresso, M. Jimenez-Palomares, N. Bozadjieva, J. P. Werneck-deCastro, M. Hatanaka, R. G. Mirmira, N. Sonenberg, M. Liu, M. A. Ruegg, M. N. Hall and E. Bernal-Mizrachi (2017). "Loss of mTORC1 signalling impairs beta-cell homeostasis and insulin processing." Nat Commun 8: 16014.

Blandino-Rosano, M., J. O. Scheys, M. Jimenez-Palomares, R. Barbaresso, A. S. Bender, A. Yanagiya, M. Liu, L. Rui, N. Sonenberg and E. Bernal-Mizrachi (2016). "4E-BP2/SH2B1/IRS2 Are Part of a Novel Feedback Loop That Controls beta-Cell Mass." Diabetes 65(8): 22352248.

Blodgett, D. M., A. Nowosielska, S. Afik, S. Pechhold, A. J. Cura, N. J. Kennedy, S. Kim, A. Kucukural, R. J. Davis, S. C. Kent, D. L. Greiner, M. G. Garber, D. M. Harlan and P. dilorio (2015). "Novel Observations From Next-Generation RNA Sequencing of Highly Purified Human Adult and Fetal Islet Cell Subsets." Diabetes 64(9): 3172-3181.

Bousquet-Antonelli, C. and J. M. Deragon (2009). "A comprehensive analysis of the La-motif protein superfamily." RNA 15(5): 750-764.

Bozadjieva, N., M. Blandino-Rosano, J. Chase, X. Q. Dai, K. Cummings, J. Gimeno, D. Dean, A. C. Powers, G. K. Gittes, M. A. Ruegg, M. N. Hall, P. E. MacDonald and E. Bernal-Mizrachi (2017). "Loss of mTORC1 signaling alters pancreatic alpha cell mass and impairs glucagon secretion." J Clin Invest 127(12): 4379-4393.

Burrows, C., N. Abd Latip, S. J. Lam, L. Carpenter, K. Sawicka, G. Tzolovsky, H. Gabra, M. Bushell, D. M. Glover, A. E. Willis and S. P. Blagden (2010). "The RNA binding protein Larp1 regulates cell division, apoptosis and cell migration." Nucleic Acids Res 38(16): 5542-5553.

Chang-Chen, K. J., R. Mullur and E. Bernal-Mizrachi (2008). "Beta-cell failure as a complication of diabetes." Rev Endocr Metab Disord 9(4): 329-343. 
Condon, K. J. and D. M. Sabatini (2019). "Nutrient regulation of mTORC1 at a glance." J Cell Sci 132(21).

Deragon, J. M. and C. Bousquet-Antonelli (2015). "The role of LARP1 in translation and beyond." Wiley Interdiscip Rev RNA 6(4): 399-417.

Efeyan, A., W. C. Comb and D. M. Sabatini (2015). "Nutrient-sensing mechanisms and pathways." Nature 517(7534): 302-310.

Fonseca, B. D., C. Zakaria, J. J. Jia, T. E. Graber, Y. Svitkin, S. Tahmasebi, D. Healy, H. D. Hoang, J. M. Jensen, I. T. Diao, A. Lussier, C. Dajadian, N. Padmanabhan, W. Wang, E. MattaCamacho, J. Hearnden, E. M. Smith, Y. Tsukumo, A. Yanagiya, M. Morita, E. Petroulakis, J. L. Gonzalez, G. Hernandez, T. Alain and C. K. Damgaard (2015). "La-related Protein 1 (LARP1) Represses Terminal Oligopyrimidine (TOP) mRNA Translation Downstream of mTOR Complex 1 (mTORC1)." J Biol Chem 290(26): 15996-16020.

Helman, A., A. L. Cangelosi, J. C. Davis, Q. Pham, A. Rothman, A. L. Faust, J. R. Straubhaar, D. M. Sabatini and D. A. Melton (2020). "A Nutrient-Sensing Transition at Birth Triggers GlucoseResponsive Insulin Secretion." Cell Metab 31(5): 1004-1016 e1005.

Hinnebusch, A. G., I. P. Ivanov and N. Sonenberg (2016). "Translational control by 5'-untranslated regions of eukaryotic mRNAs." Science 352(6292): 1413-1416.

Hong, S., M. A. Freeberg, T. Han, A. Kamath, Y. Yao, T. Fukuda, T. Suzuki, J. K. Kim and K. Inoki (2017). "LARP1 functions as a molecular switch for mTORC1-mediated translation of an essential class of mRNAs." Elife 6.

Hopkins, T. G., M. Mura, H. A. Al-Ashtal, R. M. Lahr, N. Abd-Latip, K. Sweeney, H. Lu, J. Weir, M. El-Bahrawy, J. H. Steel, S. Ghaem-Maghami, E. O. Aboagye, A. J. Berman and S. P. Blagden (2016). "The RNA-binding protein LARP1 is a post-transcriptional regulator of survival and tumorigenesis in ovarian cancer." Nucleic Acids Res 44(3): 1227-1246.

Jaafar, R., S. Tran, A. N. Shah, G. Sun, M. Valdearcos, P. Marchetti, M. Masini, A. Swisa, S. Giacometti, E. Bernal-Mizrachi, A. Matveyenko, M. Hebrok, Y. Dor, G. A. Rutter, S. K. Koliwad and A. Bhushan (2019). "mTORC1 to AMPK switching underlies beta-cell metabolic plasticity during maturation and diabetes." J Clin Invest 129(10): 4124-4137.

Jefferies, H. B., S. Fumagalli, P. B. Dennis, C. Reinhard, R. B. Pearson and G. Thomas (1997). "Rapamycin suppresses 5'TOP mRNA translation through inhibition of p70s6k." EMBO J 16(12): 3693-3704.

Jefferies, H. B., C. Reinhard, S. C. Kozma and G. Thomas (1994). "Rapamycin selectively represses translation of the "polypyrimidine tract" mRNA family." Proc Natl Acad Sci U S A 91(10): 4441-4445.

Katsumoto, K. and A. Grapin-Botton (2020). "Nutrients men-TOR beta-Cells to Adulthood." Dev Cell 54(2): 140-141.

Lahr, R. M., B. D. Fonseca, G. E. Ciotti, H. A. Al-Ashtal, J. J. Jia, M. R. Niklaus, S. P. Blagden, T. Alain and A. J. Berman (2017). "La-related protein 1 (LARP1) binds the mRNA cap, blocking elF4F assembly on TOP mRNAs." Elife 6.

Meyuhas, O. and T. Kahan (2015). "The race to decipher the top secrets of TOP mRNAs." Biochim Biophys Acta 1849(7): 801-811.

Mura, M., T. G. Hopkins, T. Michael, N. Abd-Latip, J. Weir, E. Aboagye, F. Mauri, C. Jameson, J. Sturge, H. Gabra, M. Bushell, A. E. Willis, E. Curry and S. P. Blagden (2015). "LARP1 post- 
transcriptionally regulates mTOR and contributes to cancer progression." Oncogene 34(39): 5025-5036.

Ni, Q., Y. Gu, Y. Xie, Q. Yin, H. Zhang, A. Nie, W. Li, Y. Wang, G. Ning, W. Wang and Q. Wang (2017). "Raptor regulates functional maturation of murine beta cells." Nat Commun 8: 15755.

Philippe, L., J. J. Vasseur, F. Debart and C. C. Thoreen (2018). "La-related protein 1 (LARP1) repression of TOP mRNA translation is mediated through its cap-binding domain and controlled by an adjacent regulatory region." Nucleic Acids Res 46(3): 1457-1469.

Segerstolpe, A., A. Palasantza, P. Eliasson, E. M. Andersson, A. C. Andreasson, X. Sun, S. Picelli, A. Sabirsh, M. Clausen, M. K. Bjursell, D. M. Smith, M. Kasper, C. Ammala and R. Sandberg (2016). "Single-Cell Transcriptome Profiling of Human Pancreatic Islets in Health and Type 2 Diabetes." Cell Metab 24(4): 593-607.

Shimobayashi, M. and M. N. Hall (2014). "Making new contacts: the mTOR network in metabolism and signalling crosstalk." Nat Rev Mol Cell Biol 15(3): 155-162.

Sinagoga, K. L., W. J. Stone, J. V. Schiesser, J. I. Schweitzer, L. Sampson, Y. Zheng and J. M. Wells (2017). "Distinct roles for the mTOR pathway in postnatal morphogenesis, maturation and function of pancreatic islets." Development 144(13): 2402-2414.

Stavraka, C. and S. Blagden (2015). "The La-Related Proteins, a Family with Connections to Cancer." Biomolecules 5(4): 2701-2722.

Tcherkezian, J., M. Cargnello, Y. Romeo, E. L. Huttlin, G. Lavoie, S. P. Gygi and P. P. Roux (2014). "Proteomic analysis of cap-dependent translation identifies LARP1 as a key regulator of 5'TOP mRNA translation." Genes Dev 28(4): 357-371.

Terada, N., H. R. Patel, K. Takase, K. Kohno, A. C. Nairn and E. W. Gelfand (1994). "Rapamycin selectively inhibits translation of mRNAs encoding elongation factors and ribosomal proteins." Proc Natl Acad Sci U S A 91(24): 11477-11481.

Thoreen, C. C., L. Chantranupong, H. R. Keys, T. Wang, N. S. Gray and D. M. Sabatini (2012). "A unifying model for mTORC1-mediated regulation of mRNA translation." Nature 485(7396): 109-113.

Werneck-de-Castro, J. P., M. Blandino-Rosano, D. Hilfiker-Kleiner and E. Bernal-Mizrachi (2020). "Glucose stimulates microRNA-199 expression in murine pancreatic beta-cells." J Biol Chem 295(5): 1261-1270.

Xu, Z., J. Xu, H. Lu, B. Lin, S. Cai, J. Guo, F. Zang and R. Chen (2017). "LARP1 is regulated by the $\mathrm{XIST} / \mathrm{miR}-374 \mathrm{a}$ axis and functions as an oncogene in non-small cell lung carcinoma." Oncol Rep 38(6): 3659-3667.

Ye, L., S. T. Lin, Y. S. Mi, Y. Liu, Y. Ma, H. M. Sun, Z. H. Peng and J. W. Fan (2016). "Overexpression of LARP1 predicts poor prognosis of colorectal cancer and is expected to be a potential therapeutic target." Tumour Biol 37(11): 14585-14594.

Yuan, T., S. Rafizadeh, K. D. Gorrepati, B. Lupse, J. Oberholzer, K. Maedler and A. Ardestani (2017). "Reciprocal regulation of mTOR complexes in pancreatic islets from humans with type 2 diabetes." Diabetologia 60(4): 668-678. 